\title{
ANTHROPOS - CAVASCREEN DIAGNOSTIC EXPERT SYSTEM IN ESTIMATION OF HEMODYNAMICS STATE
}

\section{Levon Dilenyan', Georgy Belkaniya², Andrew Martusevich ${ }^{\square}$ (D) Olexander Sobotnitsky ${ }^{3}$, Vladimir Kononets ${ }^{4}$, Viktor Tupitsyn ${ }^{4}$, Sergey Korshunov ${ }^{4}$}

\author{
${ }^{1}$ Privolzhsky Research Medical University, Nizhny Novgorod, Russia \\ 2 Laboratory of Medical Expert Systems "Antropos Systems Lab.", Vinnitsa, \\ Ukraine \\ ${ }^{3}$ Institute of Medical Technology and Equipment ITAM. Zabrze, Poland \\ ${ }^{4}$ R.E. Alekseev Nizhny Novgorod State Technical University, Nizhny \\ Novgorod, Russia
}

\section{cryst-mart@yandex.ru}

A BSTRACT - An innovative diagnostic complex has been created that allows a systematic approach to analyzing the performance of various blood circulation sections - the heart itself and its regulation (ECG), central and peripheral hemodynamics (according to the reogramme), skin circulation (up to 382 indicators). This allows for a qualitatively different level to assess the state of healthy individuals and patients in the prone and standing position, and to use these data for the diagnosis and correction of treatment.

KEY W ORDS - Anthropos-cavascreen, hemodynamics, diagnostics.

The most important factor in the development of medicine are innovative methods in the diagnostics and support of human health. This possibility is realized on the basis of the system assessment of hemodynamics according to the complex technique of tetrapolar thoracic and regional rheography developed by us with the use of the laboratory of medical expert systems Anthropos systems lab. (Vinnitsa, Ukraine) and the Institute of Medical Technology and Equipment ITAM. Zabrze, Poland)) hardware-software complex ANTHROPOS-CAVASCREEN (Fig. 1).

The research was carried out within the framework of the international project on the European Fund EUREKA (Eureka EU 2939 project dated 31.1.2003). On the basis of the experimental and clinical materials obtained by us, the optimal algorithm for the analysis of the thoracic reocardiocycle and peripheral rheograms is formed and justified.

In our complex the system and multilevel complex assessment based on basic laws of functioning of cardiovascular system, including features of regula-

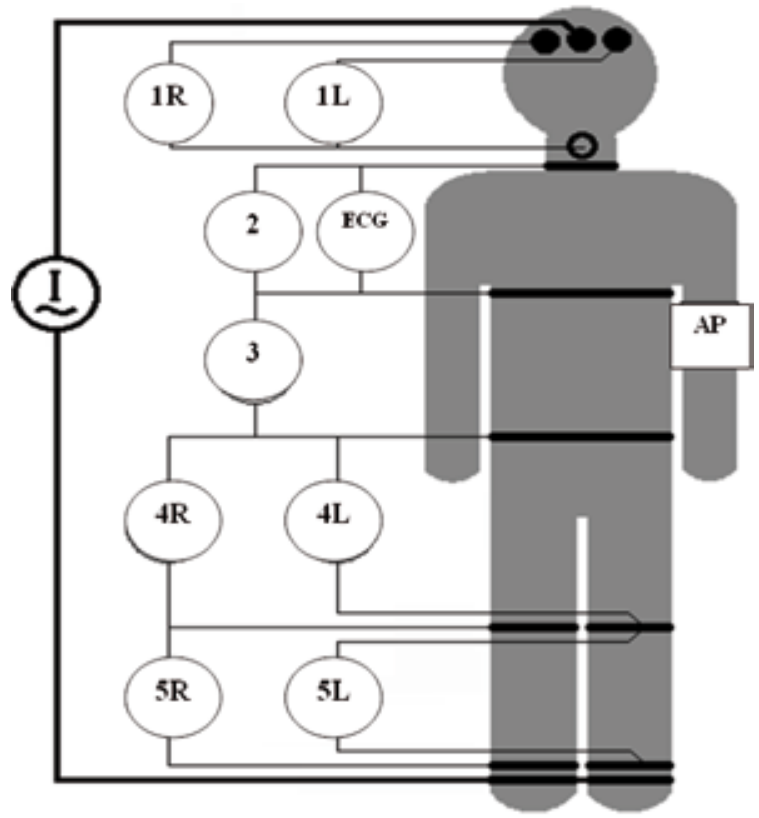

Fig.1. Scheme of tetrapolar superposition of current (I) electrodes and potential electrodes for rheographic registration of hemodynamic parameters. Designations on the connected system of potential electrodes: 1-head, 2-chest, 3-abdomen, 4-pelvis-thigh, 5-Shin; $R$-right, $L$ - left. AP-cuff measurement of blood pressure, ECG-electrocardiogram

tion on hydrostatic (gravitational) factor of blood circulation at the person in standing position is used. The complex of direct and settlement hemodynamic indicators is analyzed. To analyze and evaluate the state of blood circulation, 130 measured and calculated indicators in the standing and lying position are used, as well as 102 related indicators with respect to their values according to the mandatory conditions of the study (standing-lying). Additionally, 20 indicators are determined for the samples standing and lying down with breath retention. In total, according to the conditions of the anthropophysiological study, up to 382 indicators are used in the diagnostic assessment of hemodynamic support of the somatic state.

\section{GENERAL CHARACTERISTICS}

OF COMPONENTS OF ANTHROPOSCAVASCREEN:

- Implementation of multichannel synchronous registration of basic and differential rheograms 
reflecting pulse blood flow of the brain on the left and right, lungs, abdominal hemodynamics, blood circulation of the pelvis and lower extremities (shins) symmetrically on the left and right.

- An electrocardiogram is recorded at the same time.

- Measurement of the basic impedance characteristic of the investigated hemodynamic blocks.

- Study of cutaneous blood flow and circulatory thermoregulation by measuring the electrical conductivity of the skin by a modified Nakatani technique.

- Cuff blood pressure measurement.

- Calculation of the main hemodynamic parameters.

- On the basis of anthropophysiologically oriented multiparameter analysis of the state of blood circulation in the standing and lying positions, a package of output documentation is automatically formed - a complete Protocol and a diagnostic conclusion.

- In the diagnostic conclusion in verbal and graphic form the system characteristic of a condition of cardiovascular system on the main hemodynamic mechanisms (volume - capacity - the pump function - a blood flow), as a whole and on the main blocks and components of blood circulation is given, and also all revealed hemodynamic syndromes separately on position of a body standing and lying are described.

\section{GENERAL FEATURES AND PURPOSE OF ANTHROPOS-CAVASCREEN TECHNOLOGY}

The hardware-software complex and expert system is designed to diagnose the state of the cardiovascular system. Instrumental techniques used in the system (multichannel tetrapolar thoracic and regional rheography, electrometry of the skin by electrical conductivity, oscillometric measurement of blood pressure) are non-invasive and do not cause inconvenience to the patient.

The diagnostic concept of diagnostic expert system Anthropos-Cavascreen for the cardiovascular system on the basis of anthropological approach is considered as an anatomical and functional integrity of natural biological quality of man (bipedalism) and directly associated value of the gravitational factor in the circulation.

Computer hardware and software complex Anthropos-Cavascreen allows to simultaneously measure the parameters of the state of 8 hemodynamic blocks. Assessment of circulation in separate functional units (blood pressure, blood volume, the pumping function of the left and right heart, small and large circle circulation, arterial and venous blood circulation of the head left and right, abdomen, pelvis, thigh and lower leg left and right) is not isolated, but comprehensively and systematically in the context of the general state of blood circulation and other functional blocks. The study is carried out in two typical variants for man as an upright creature body positions (standing and lying). This takes into account the hemodynamic parameters of both postural conditions, as well as the associated characteristic of the condition standing/ lying.

When analyzing the data obtained for each position of the body, we use sex and age ratios of the absolute values of direct and derived hemodynamic parameters, as well as percentages of these values in standing relative to their size in the supine position (standard model state).

The expert system Anthropos-Cavascreen on the basis of multiparameter comparison of the measured hemodynamic parameters with the normative model and the use of the algorithm of multilevel criteria and syndrome analysis forms an individual state model, which is described by the diagnostic conclusion. In conclusion, only syndromes are presented in descriptive (verbal) and graphic form, as functionally and clinically significant circulatory conditions, the group characteristic of which does not correspond to the normative model, but coincides with the hemodynamic profile of the model of circulatory disorders.

The expert system Anthropos-Cavascreen allows to carry out screening and dynamic diagnostics of cardiovascular system at early stages of violations of its condition, helps the doctor in decision-making on correction of the revealed changes, and also allows to monitor efficiency of the carried-out treatment.

Given the importance of optimal hemodynamic support of any somatic condition in the full range of bealth-illness-disease, diagnostic information obtained with the help of Anthropos-Cavascreen" is useful in the pre-medical and medical support of healthy, pregnant, patients of any nosological profile.

Computer hardware and software complex Anthropos-Cavascreen is designed for functional diagnostics departments, polyclinics, hospitals and institutions of health profile, insurance medicine, as well as to provide a workplace for a family doctor.

\section{CONCLUSION}

The hardware and software expert system ANTHROPOS-CAVASCREEN allows a systematic approach to the analysis of indicators of various departments of blood circulation: the heart and its regulation (ECG), central and peripheral hemody- 
namics (rheogram), skin circulation (up to 382 indicators). The use of the algorithm of multilevel criteria and syndrome analysis reveals an individual state model, including syndromes of functionally and clinically significant circulatory conditions, the group with the hemodynamic profile of the model of circulatory disorders. This allows to fully assess the condition of the examined persons in the lying and standing positions. Studies performed in more than 2,000 patients have shown the value of using these data for diagnosis and treatment correction. The research methodology of the hardware and software complex is protected by an international patent.

\section{REFERENCES}

1. Belkaniya G.S., Dilenyan L.R., BagriI A.S., RYzhakov D.I. ET AL. Anthropological approach in the diagnostic evaluation of the cardiovascular system // Medical almanac. - 2013. - № 4. - P. 108-114.

2. Belkaniya G.S., Dilenyan L.R., Bagri A.S., RYZHAKov D.I. ET AL. Features of methodological support of anthropophysiological diagnostics of the cardiovascular system // Medical almanac. - 2013. №6. - P. 208-214.
3. Belkaniya G.S., Dilenyan L.R., BagriI A.S. RYZhakov D.I. ET AL. Anthropological approach to the formation of a diagnostic scale for hemodynamic parameters // Medical almanac. - 2014. - № 2. - P. $152-156$.

4. Belkaniya G.S., Dilenyan L.R., Bagri A.S., RyzhaKov D.I. ET AL. Anthropological approach in the system algorithm criterion analysis state of the cardiovascular system // Medical almanac. - 2014. № 5. - P. 170-174.

5. Dilenyan L.R., Belkaniya G.S., BagriI A.S., RyzHAKov D.I. ET AL. Syndromic analysis of the cardiovascular system // Medical almanac. - 2015. № 1. - P. 125-130.

6. Dilenyan L.R., Martusevich A.K., Bocharin I.V., BelKaniYa G.S. Typological structure of hemodynamics in age scale // Archiv Euromedica. - 2019. - Vol. 9, №2. - P. 65-67.

7. Sayenko D.G., Artamonov A.A., Kozlovskaya I.B. Characteristics of postural corrective responses before and after long-term spaceflights / / Human Physiol. -2015. -Vol. 37, № 5. - P. 91-99.

8. Zampieri, P. Medicine, Evolution, and Natural Selection: an historical overview // The Quarterly Review of Biology. - 2009. -Vol. 4. - P. 333-355. 